Chirurg 2018 $89: 50-55$

https://doi.org/10.1007/s00104-017-0504-2

Online publiziert: 13 . September 2017

(c) Der/die Autor(en) 2017. Dieser Artikel ist eine Open-Access-Publikation.

CrossMark

\author{
J. Jakob ${ }^{1} \cdot$ A. Gerres ${ }^{2} \cdot$ U. Ronellenfitsch ${ }^{3} \cdot$ L. Pilz $^{2} \cdot$ M. Wartenberg ${ }^{4} \cdot$ B. Kasper ${ }^{5} \cdot$ \\ H.-R. Raab ${ }^{6}$ P. Hohenberger ${ }^{1}$ \\ 'Spezielle Chirurgische Onkologie und Thoraxchirurgie, Chirurgische Klinik, Universitätsmedizin \\ Mannheim, Universität Heidelberg, Mannheim, Deutschland \\ ${ }^{2}$ Medizinische Fakultät Mannheim, Universität Heidelberg, Heidelberg, Deutschland \\ ${ }^{3}$ Klinik für Gefäßchirurgie, Universitätsklinikum Heidelberg, Heidelberg, Deutschland \\ ${ }^{4}$ Das Lebenshaus, Wölfersheim, Deutschland \\ ${ }^{5}$ Interdisziplinäres Tumorzentrum Mannheim, Universitätsmedizin Mannheim, Universität Heidelberg, \\ Heidelberg, Deutschland \\ ${ }^{6}$ Universitätsklinik für Allgemein- und Viszeralchirurgie, Klinikum Oldenburg, Oldenburg, Deutschland
}

\title{
Behandlung retroperitonealer Sarkome in Deutschland
}

\section{Eine Umfrage der Deutschen Gesellschaft für Allgemein- und Viszeralchirurgie, der Deutschen Interdisziplinären Studiengruppe Sarkome und der Patientenorganisation Das Lebenshaus}

\section{Zusatzmaterial online}

Die Online-Version dieses Beitrags (https:// doi.org/10.1007/s00104-017-0504-2) enthält den Umfragebogen inkl. Auswertung. Beitrag und Zusatzmaterial stehen Ihnen im elektronischen Volltextarchiv auf http:// www.springermedizin.de/der-chirurg zur Verfügung. Sie finden das Zusatzmaterial am Beitragsende unter "Supplementary Material".

\section{Hinführung zum Thema}

Retroperitoneale Sarkome sind seltene Tumore. In Deutschland existieren keine zentralisierte Behandlung und keine nationale Behandlungsleitlinie für retroperitoneale Sarkome. Außerdem liegen keine Daten zur Behandlungsstrategien und -erfolgen aus Registern vor. Ziel dieser Studie ist die Erfassung der primären Diagnostik und Therapie von retroperitonealen Sarkomen in Deutschland durch eine Onlineumfrage.

\section{Hintergrund}

Die Inzidenz von retroperitonealen Sarkomen (RPS) beträgt ca. 0,5 bis 1:100.000
$[1,2]$. Abhängig vom histologischen Subtyp besteht eine deutliche Variabilität im klinischen Verlauf. Während z. B. Patienten mit gut differenzierten Liposarkomen fast nie Fernmetastasen entwickeln, jedoch auch nach vollständiger Resektion ein deutliches Risiko für - bisweilen lebensbedrohliche - Lokalrezidive haben, besteht $z$. B. bei Patienten mit retroperitonealen Leiomyosarkomen auch eine hohes Risiko für pulmonale oder hepatische Metastasen [3].

Der allgemein akzeptierte Therapiestandard retroperitonealer Sarkome ist trotz dieser Unterschiede die vollständige chirurgische Resektion [4, 5]. Die empfohlene - mitunter kontrovers diskutierte - Operationsstrategie ist die retroperitoneale Kompartmentresektion, d. h. die Mitresektion nicht infiltrierter, jedoch adhärenter Organe (z. B. Kolon und Niere) $[6,7]$. Auch wenn Kohortenstudien die Effektivität einer präoperativen Strahlentherapie nahelegen, ist ein Überlebensvorteil hierdurch nicht nachgewiesen und wird derzeit in einer randomisierten Studie der EORTC überprüft (NCT01344018) [8, 9]. Systemische The- rapien gehören nach aktuellem Kenntnisstand nicht zur Standardtherapie, können jedoch in Einzelfällen zur Anwendung kommen, ggf. auch in Kombination mit einer regionalen Hyperthermie [10].

Die o. g. Therapieempfehlungen sind Ergebnisse von intensiven Diskussionen der Transatlantischen Arbeitsgruppe Retroperitoneale Sarkome (TARPS) sowie der NCCN (National Comprehensive Cancer Network, USA) und der ESMO (European Society for Medical Oncology). Für Deutschland liegen derzeit keine Leitlinien vor, eine S3-Leitlinie Sarkome ist in Vorbereitung. Weiterhin existieren in Deutschland (noch) keine Daten aus klinischen oder epidemiologischen Krebsregistern, die Rückschlüsse auf die Behandlung bzw. Behandlungsergebnisse ermöglichen. Auch vonseiten des Robert Koch-Instituts wird derzeit lediglich die Inzidenz von Tumoren der ICD C48 ohne weitere Differenzierung oder Angabe der Überlebensraten berechnet.

Zur Abschätzung der gegenwärtigen Versorgungsstrukturen von Patienten mit retroperitonealen Sarkomen wur- 
de daher diese Analyse initiiert. Der Fragenkatalog wurde im Konsens mit der Chirurgischen Arbeitsgemeinschaft Onkologie der Deutschen Gesellschaft für Allgemein- und Viszeralchirurgie (CAO-V der DGAV, www.dgav.de), der Patientenorganisation Das Lebenshaus e. V. (www.daslebenshaus.org) und der deutschen Sarkomstudiengruppe (German Interdisciplinary Sarcoma Group (GISG), www.gisg.de) entworfen.

\section{Methodik}

Der Fragenkatalog wurde gemeinsam mit CAO-V, GISG und Das Lebenshaus e. V. entwickelt und umfasst insgesamt 29 Fragen. Im ersten Teil der Umfrage wurde nach dem diagnostischen Vorgehen (z. B. Art der Bildgebung, Durchführung einer Biopsie) gefragt. Ein zweiter Teil befasste sich mit der Operationsstrategie (z. B. Resektion von Nachbarorganen, systematische Lymphadenektomie, Kriterien der Irresektabilität) sowie mit multimodalen Therapieansätzen (z. B. Anteil der Patienten mit Strahlen- oder Chemotherapie). Allgemeine Fragen nach Charakteristika des Zentrums (z. B. Universitätsklinik vs. andere, Anzahl der RPS-Patienten pro Jahr) sowie nach Fortbildungen, Infomaterial und einem zentralen DGAV-Register (StuDoQ) schlossen die Umfrage ab. Der Fragenkatalog ist in der OnlineVersion dieses Beitrags im Appendix einsehbar („Supplementary Material“).

Die Umfrage wurde als Onlinesurvey entworfen. Design und elektronischer Versand wurden mit SoSci Survey durchgeführt (www.soscisurvey.de). SoSciSurvey ist eine für akademische Umfragen kostenlose Online-Umfrageplattform, die eine verschlüsselte Datenübermittlung und eine anonymisierte Auswertung mit Datenspeicherung in Deutschland ermöglicht. Chirurgische Abteilungen sind am ehesten primäre Ansprechpartner von Patienten mit nicht metastasierten retroperitonealen Sarkomen. Daher adressierten wir alle leitenden Ärzte (viszeral-)chirurgischer Abteilungen in Deutschland ( $n=976$ ). Die entsprechenden Adressen wurden dem deutschen Krankenhausverzeichnis entnommen und vor Versenden der Umfrage aktualisiert. Eine Einladung zur Teilnahme an der Umfrage wurde gemeinsam mit einem Link zum Fragebogen elektronisch versandt. Regelmäßig wurde elektronisch an die Teilnahme erinnert. Die Umfrage wurde zwischen Dezember 2015 und August 2016 durchgeführt.

Die statistische Analyse erfolgte zunächst deskriptiv. Häufigkeit und Verteilung der Antworten wurden absolut und prozentual angegeben. Gruppenvergleiche wurden mit dem Chi-Quadrat bzw. dem exakten Fisher-Test durchgeführt. Geografische Unterschiede wurden durch Gruppierung in Nord (Bundesländer Bremen, Hamburg, SchleswigHolstein, Niedersachsen), Ost (Berlin, Brandenburg, Mecklenburg-Vorpommern, Sachsen, Sachsen-Anhalt), West (Hessen, Nordrhein-Westfalen, Rheinland-Pfalz, Saarland) und „Süd“ (BadenWürttemberg, Bayern) geprüft. Aufgrund unterschiedlicher Definitionen in den Bundesländern konnte keine Einteilung in Abteilungen aus Krankenhäusern der Grund- und Regelversorgung bzw. Maximalversorgung erfolgen. Stattdessen stratifizierten wir alle Ergebnisse zwischen Abteilungen, die weniger als 10 RPS Patienten jährlich behandeln, und solchen, die 10 RPS oder mehr behandeln. Benutzt wurde die Statistik-Software SPSS (Version 22). Das Signifikanzniveau wurde auf $p=0,05$ festgelegt.

\section{Ergebnisse}

An der Umfrage nahmen 191 der 976 adressierten Abteilungen teil. Das entspricht einer Rücklaufquote von $20 \%$. Keine RPS zu behandeln gaben 24 von 191 (13\%) Teilnehmern an. Den Fragebogen füllten 135 von 191 (71\%) Teilnehmern vollständig aus. Von 38 nahmen 27 (71 \%) der chirurgischen Abteilungen an Universitätskliniken an der Umfrage teil und beantworteten jeweils alle Fragen vollständig. Sämtliche Ergebnisse können mit Angabe der absoluten und prozentualen Häufigkeit der Antworten im Anhang eingesehen werden. Hier erfolgt zunächst eine Zusammenfassung der Ergebnisse aller Teilnehmer. Im Weiteren werden Unterschiede zwischen Krankenhäusern mit mehr als 10 retroperitonea- len Sarkomen und anderen Krankenhäusern diskutiert.

Im Rahmen der Diagnostik werden sowohl Kernspintomographie als auch Computertomographie regelhaft als abdominelle Bildgebung eingesetzt (bei $62 \%$ der Teilnehmer beides). Bei über $90 \%$ der Teilnehmer erfolgt der Ausschluss von Lungenmetastasen regelhaft durch ein thorakales Computertomogramm. Bemerkenswert ist, dass bei $17 \%$ regelhaft ein PET/CT durchgeführt wird. Eine Tumorbiopsie vor Therapiebeginn wird in lediglich $53 \%$ der Kliniken regelhaft vorgenommen. In 28 Krankenhäusern existiert ein spezielles Sarkomboard, ansonsten werden RPS-Fälle in einem anderen Tumorboard besprochen.

Die üblicherweise gewählte Resektionsstrategie ist in $82 \%$ der Kliniken eine abdominelle Kompartmentresektion i.S. einer Multiviszeralresektion. Etwas weniger (69\%) würden dabei auch nicht infiltrierte, lediglich adhärente Organe entfernen. Dass dabei jeweils zwei oder mehr Organe reseziert werden, gaben $64 \%$ der Teilnehmer an. Eine systematische Lymphadenektomie führen regelhaft $39 \%$ der Teilnehmer der Umfrage durch. Als häufigste Irresektabilitätskriterien wurden angesehen: die Infiltration der Mesowurzel (75\%), der paravertebralen Muskulatur und Neuroforamina (51 \%) sowie die Notwendigkeit der Resektion und Rekonstruktion von Aorta (22\%) oder V. cava (11\%). In $71 \%$ der Krankenhäuser würde auch in Gegenwart einer einzelnen Lungenmetastase die Primärtumorresektion durchgeführt. Die Rate an revisionsoder interventionspflichtigen Komplikationen im eigenen Krankengut wird von $83 \%$ der Teilnehmer auf bis zu $10 \%$ geschätzt, die Eingriffsmortalität von $82 \%$ der Teilnehmer auf bis zu $2 \%$.

Eine zusätzliche Strahlentherapie wird in $26 \%$ der Krankenhäuser regelhaft ( $>50 \%$ der Fälle) durchgeführt. Üblicherweise wird diese in $86 \%$ der Kliniken postoperativ durchgeführt. Insgesamt gaben $22 \%$ der Kliniken an, dass in $>50 \%$ der RPS Patienten eine zusätzliche Chemotherapie gegeben wird. In $58 \%$ der Krankenhäuser erfolgt die Chemotherapie üblicherweise postoperativ. 
Chirurg 2018·89:50-55 https://doi.org/10.1007/s00104-017-0504-2

@ Der/die Autor(en) 2017. Dieser Artikel ist eine Open-Access-Publikation.

J. Jakob · A. Gerres · U. Ronellenfitsch · L. Pilz · M. Wartenberg · B. Kasper · H.-R. Raab · P. Hohenberger

Behandlung retroperitonealer Sarkome in Deutschland. Eine Umfrage der Deutschen Gesellschaft für Allgemein- und Viszeralchirurgie, der Deutschen Interdisziplinären Studiengruppe Sarkome und der Patientenorganisation Das Lebenshaus

\section{Zusammenfassung}

Einleitung. Retroperitoneale Sarkome sind seltene bösartige Tumore. In Deutschland existieren keine zentralisierte Behandlung und keine nationale Behandlungsleitlinie für retroperitoneale Sarkome. Ziel dieser Studie ist die Erfassung der primären Diagnostik und Therapie von retroperitonealen Sarkomen in Deutschland durch eine Onlineumfrage. Methodik. Durchführung einer Umfrage an allen chirurgischen Abteilungen der Universitätskliniken und Krankenhäusern der Maximal- und Regelversorgung $(n=976)$. Erfragt wurden Bildgebung, Biopsie, multimodale Therapie und operative Verfahren. Es handelte sich um eine plattformgestützte Onlineumfrage.
Ergebnisse. Die Rücklaufquote betrug $20 \%$. Lediglich 11 der antwortenden Kliniken behandeln mehr als 10 Patienten im Jahr. Zur Diagnostik werden generell Schnittbildverfahren eingesetzt, ein Ausschluss von pulmonalen Metastasen erfolgt in $96 \%$ der Kliniken durch eine Computertomographie. In $54 \%$ der Abteilungen wird vor einer Resektion regelhaft eine Tumorbiopsie durchgeführt. Das hauptsächlich eingesetzte Resektionsverfahren stellt die retroperitoneale Kompartmentresektion mit $85 \%$ dar. Eine systematische Lymphadenektomie erfolgt in $40 \%$ der Abteilungen. Eine perioperative Strahlen- oder Chemotherapie wird in $27 \%$ bzw. $22 \%$ der Kliniken regelhaft durchgeführt.
$84 \%$ würden die Durchführung eines Workshops über die Diagnostik und Therapie von Weichgewebesarkomen begrüßen. Schlussfolgerung. Es zeigt sich in Diagnostik und Therapie kein einheitliches Vorgehen bei retroperitonealen Sarkomen, was an der niedrigen Inzidenzrate und Behandlungsfrequenz sowie dem Fehlen einer nationalen S3Leitlinie liegen könnte.

Schlüsselwörter

Umfrage - Retroperitoneales Sarkom - Behandlungsstandard $\cdot$ Multiviszeralresektion . Multimodale Therapie

\section{Treatment of retroperitoneal sarcoma in Germany. Results of a survey of the German Society of General and Visceral Surgery, the German Interdisciplinary Sarcoma Study Group and the advocacy group Das Lebenshaus}

\begin{abstract}
Objective. Retroperitoneal sarcomas (RPSs) are rare cancers with some variability in clinical and histopathological presentation. In Germany, general treatment strategies of retroperitoneal sarcoma are unknown since centralized registries do not exist. The objective of this survey was to access the medical care of RPS patients in Germany. Methods. In cooperation with the German Society of General and Visceral surgery, the German Interdisciplinary Sarcoma Study Group and the patient advocacy group Das Lebenshaus we designed an online survey assessing diagnostic and treatment strategies (e. g. performance of tumor biopsies,
\end{abstract}

administration of multimodal therapies and surgical strategy). All departments for general and visceral surgery in Germany were addressed $(n=976)$.

Results. Responses were received from 191 of 976 departments. Only 11 surgical departments treat more than 10 RPS patients per year. A multidisciplinary sarcoma board exists in 19 hospitals. Staging is generally performed by cross-sectional imaging. In $54 \%$ of the departments pretreatment tumor biopsy is a standard procedure. Surgery is performed as compartment resection in $85 \%$ of the departments. A systematic lymph node dissection is done in $40 \%$. Adjuvant radio- or chemotherapy is performed as a standard treatment in $27 \%$ and $22 \%$ departments, respectively.

Conclusion. The survey demonstrates a large heterogeneity in RPS diagnostic and treatment strategies. Dedicated education programs and centralized treatment strategies are warranted to improve the standard of care.

Keywords

Survey $\cdot$ Retroperitoneal sarcoma $\cdot$ Standard of care - Multivisceral resection - Multimodal therapy
$32 \%$ der Teilnehmer gaben an, im letzten Jahr keine Patienten mit retroperitonealen Sarkomen behandelt zu haben, $47 \%$ bis zu fünf Patienten, $13 \%$ sechs bis zehn und $7 \%$ mehr als zehn Fälle. Die Behandlungsfrequenz hatte dabei z. T. signifikanten Einfluss auf die diagnostischen und therapeutischen Strategien (•Tab. 1). So werden in Häusern mit höherer Behandlungsfrequenz deutlich häufiger prätherapeutische Biopsien entnommen, eher Kompartmentresekti- onen durchgeführt und seltener systematische Lymphadenektomien. Aus der geografischen Stratifikation ergaben sich hingegen keinerlei Hinweise auf statistisch signifikante regionale Unterschiede in Diagnostik und Behandlung von RPS.

\section{Diskussion}

Derzeit existieren in Deutschland im Gegensatz zu anderen Staaten wie z. B. Großbritannien weder eine zentrali- sierte Behandlung noch eine nationale Behandlungsleitlinie für retroperitonealer Sarkome. Diese Umfrage sollte daher Daten zu aktuellen Behandlungsstrategien erheben. Die Rücklaufquote der Umfrage betrug $20 \%$, wodurch es aufgrund fehlender Antworten zu einer Ergebnisverzerrung gekommen sein kann. Im Vergleich zu einer ähnlichen Umfrage der DGVS zur Behandlung von Pankreaskarzinomen (13\%) war der Rücklauf jedoch deutlich höher (mit 
Tab. 1 Diagnostik und Therapie stratifiziert nach Anzahl der behandelten Patienten pro Jahr

$<10$ Patienten im Mehr als $10 \mathrm{~Pa}$ -

Jahr $(n=123) \quad$ tienten im Jahr

$(n=11)$

Wird bei Ihnen regelhaft vor Therapiebeginn eine Biopsie des Tumors durchgeführt? ${ }^{\mathrm{a}}$

a) Ja

b) Nein

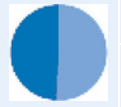

$51 \%$

$49 \%$

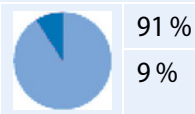

Welche Operationsstrategie wählen Sie üblicherweise?

a) + b)

c)

Enukleation oder marginale Resektion

Abdominelle Kompartmentresektion

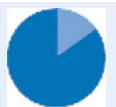

$15 \%$

$85 \%$

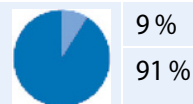

Wann erfolgt üblicherweise eine Resektion von Nachbarorganen? ${ }^{\mathrm{a}}$

a) Nur bei eindeutiger Infiltration

b) Auch bei Adhärenz
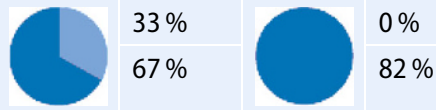

Führen Sie regelhaft eine systematische Lymphadenektomie durch?

a) Ja

b) Nein

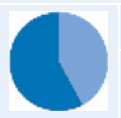

$42 \%$

$58 \%$

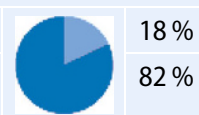

Würden Sie eine Tumorresektion bei Vorliegen einer einzelnen Lungenmetastase durchführen?
a) Regelhaft
b) + c) Selten oder nie

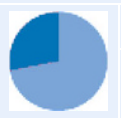

$72 \%$

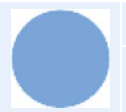

$100 \%$

$0 \%$

Wie häufig wird bei Ihnen eine zusätzliche Strahlentherapie durchgeführt?
a) + b) $\quad<50 \%$
c) + d) $\quad 51-100 \%$

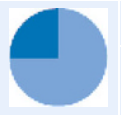

$75 \%$

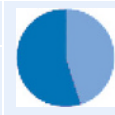

$45 \%$

$55 \%$

${ }^{a}$ Es bestand ein signifikanter Unterschied bei Tumorbiospien $(p=0.01)$ und der Resektion von Nachbarorganen ( $p=0,03$, exakter Test nach Fisher).

$20 \%$ und entsprach derjenigen, die nordamerikanische Kollegen bei einer Umfrage von Mitgliedern der MSTS (Musculosceletal Tumor Society) über die Nachsorge von Sarkomen erreichten $[11,12]$. Die Rücklaufquote kann daher als absolut angemessen eingestuft werden. Diskutiert werden sollen im Folgenden neben auffälligen Einzelergebnissen auch die Unterschiede zwischen Abteilungen mit niedriger und hoher Behandlungsfrequenz.

Biopsien werden regelhaft nur von gut der Hälfte der Kliniken durchgeführt. Die Indikation zur Biopsie ergibt sich aus wesentlichen Differenzialdiagnosen (z. B. Lymphomen), die eine operative Therapie verbieten, sowie der Tatsache, dass nur nach histologischer Sicherung eine multimodale Therapie geplant werden kann $[13,14]$. Es ist weiterhin fraglich, ob eine Multiviszeralresektion ohne vorherige histologische Sicherung in An- betracht der möglichen Morbidität auch als alleinige Therapie vertretbar ist. Befürchtungen einer Tumorzellverschleppung mit evtl. erhöhtem Rezidivrisiko mögen der Auslassung von Biopsien zugrunde liegen. In der Literatur finden sich diesbezüglich jedoch mehrere Studien, die keine relevante Erhöhung des Lokalrezidivrisikos durch Stanzbiopsien von Sarkomen nachweisen - explizit bei retroperitonealen Sarkomen-, auch ohne Ausschneiden des Biopsiekanals $[15,16]$. Die Verwendung von Koaxialnadeln zur Führung der Biopsienadel wird jedoch empfohlen.

Hauptsächlich angewandte Operationsstrategie ist die Kompartmentresektion, d. h. ggf. eine Multiviszeralresektion auch mit Entfernung solcher Organe, die nur adhärent, jedoch nicht eindeutig infiltriert sind [6]. Theoretisch setzt sich hier der internationale Trend einer im Zweifel aggressiveren chirurgischen The- rapie durch, da mit dieser Vorgehensweise das Risiko lebensbedrohlicher Lokalrezidive vermieden werden kann [3]. Die hiermit einhergehende Eingriffsmorbidität wird ungefähr in Höhe derjenigen aktueller berichteter Kohorten eingeschätzt (10-30 \% Grad III nach Clavien-Dindo), ebenso wie der Anteil von R2-Resektionen (ca. $5 \%$ ). In $39 \%$ der chirurgischen Abteilungen wird zusätzlich zur Resektion des Primarius regelhaft eine systematische Lymphadenektomie durchgeführt. Erstaunlich ist diese hohe Zahl vor allem in Anbetracht der extrem seltenen Lymphknotenmetastasen der häufigsten retroperitonealen Sarkome (Lipo- und Leiomyosarkome) und der völlig unklaren anatomisch-chirurgischen Ausdehnung einer solchen Lymphknotendissektion [17]. Es ist anzunehmen, dass reflexhaft chirurgisch-onkologische Prinzipien der Karzinomchirurgie auf Sarkome übertragen werden, ohne dass der Sinn dieser Maßnahmen im Einzelfall geprüft wird.

Für eine Mehrzahl der Teilnehmer der Umfrage stellt die vollständige chirurgische Resektion die Standardtherapie retroperitonealer Sarkome dar. Multimodale Therapieansätze wie eine präoperative Strahlen- oder Chemotherapie werden in über $50 \%$ der Fälle von $27 \%$ bzw. $22 \%$ der Teilnehmer eingesetzt, wobei eine Mehrzahl der Abteilungen eher eine postoperative Strahlen- oder Chemotherapie einsetzen würde. Dies ist insofern ungewöhnlich, als z. B. eine postoperative Strahlentherapie eine deutlich höhere Morbidität aufweist. Im Gegensatz zu einer präoperativen Strahlentherapie kann postoperativ der Tumor nicht als sein eigener Platzhalter wirken, und strahlenempfindliche Organe wie z. B. der Dünndarm kommen regelhaft im ehemaligen Tumorbett zu liegen. Hierdurch ist die Strahlentherapie kaum in voller Dosis applizierbar [18]. Wenn also bestrahlt werden sollte, ist eine präoperative Therapie weitaus sinnvoller.

Bei den multimodalen Therapien zeigt sich ebenso wie bei Tumorbiopsien und Resektionsstrategie ein deutlicher Unterschied zwischen den Abteilungen in Abhängigkeit von der Behandlungsfrequenz. Chirurgische Kliniken mit einer höheren Fallzahl (mehr als 10 RPS/Jahr) 
führen deutlich häufiger präoperative Biopsien durch, resezieren retroperitoneale Sarkome eher als Kompartmentresektion ohne Lymphadenektomie und setzen auf präoperative Strahlen- oder Chemotherapien (•Tab. 1). Dabei haben Erfahrung und Expertise sicherlich Einfluss auf die Einhaltung dieser diagnostischen bzw. therapeutischen Standards. Eine Differenzierung zwischen "low“ und "high volume" Zentren auf der Basis eigener Fallzahlangaben ist per se mit Unsicherheiten behaftet. Dennoch kann davon ausgegangen werden, dass Chefärzte einschätzen können, ob sie nur einzelne oder mehr als 10 bzw. 20 Patienten im Jahr mit RPS behandeln. Eine prinzipielle Unterscheidung erscheint daher gerechtfertigt.

Aus Versorgungsforschungsprojekten ist gut bekannt, dass bei Sarkomen Verstöße gegen die gute klinische Praxis insbesondere zu Beginn der Therapie nicht mehr reparabel sind: fehlende Diagnostik und insuffiziente chirurgischen Therapien führen nicht nur zu erhöhten Behandlungskosten, sondern auch zu einem schlechteren Überleben der Sarkompatienten [19]. Daher ist der hier explizit ausgesprochene Wunsch nach mehr Weiterbildung (84\%, ein DGAV-Seminar "Retroperitoneale Sarkome“ ist für 2017 geplant), Zusendung von Informationsmaterial für Patienten (75 \%) und einem nationalen Register (94\%, z. B. StuDoQ) zu begrüßen.

Der BegriffSarkomzentrum an sich ist nicht geschützt. Vonseiten der Deutschen Krebsgesellschaft besteht derzeit lediglich die Möglichkeit, einen Schwerpunkt (S2) „Maligne Tumoren des muskuloskeletalen Systems“ innerhalb eines onkologischen Zentrums einzurichten, der die Behandlung von Sarkomen einschließt. In Vorbereitung ist die Einrichtung eines Moduls „Zentren für Tumoren/Sarkome des Weichgewebes“. Bis Ende 2017 sollen die Voraussetzungen hierfür definiert werden. Fraglich ist, ob eine weitergehende Zertifizierung von Seiten der DGCH oder DGAV notwendig ist. Auch wenn die wichtigste Therapie von retroperitonealen Sarkomen die vollständige Resektion ist, erfordern Diagnostik und Therapie eine interdisziplinäre Expertise, um erfolgreich zu sein. Das schließt z. B. die Bildung eines Sarkomboards ein, das ak- tuell nur in ca. $15 \%$ der antwortenden Kliniken existiert. Die Diskussion von Sarkompatienten in allgemeinen Tumorboards erscheint zwar prinzipiell sinnvoll, wenn kein Sarkomboard existiert. Die Expertise von Kollegen, die sich regemäßig mit Sarkompatienten beschäftigen, ist hierdurch aber sicher nicht zu ersetzen. Als Leitfaden zur Entscheidungsfindung innerhalb eines Sarkomboards wird in Zukunft die S3-Leitlinie „Adulte Weichgewebesarkome“ dienen, die sich derzeit in Vorbereitung findet. Von chirurgischer Seite ist aber sicher die Einrichtung eines StuDoQ Registers sinnvoll. Hieraus kann sich am ehesten ein chirurgisches Behandlungs- und Forschungsnetzwerk zur Verbesserung der Versorgungsqualität von Patienten mit retroperitonealen Sarkomen entwickeln. Die Ergebnisse der Umfrage signalisieren zumindest eine hohe Bereitschaft, $\mathrm{zu}$ einem StuDoQ-Register „Retroperitoneale Sarkome“ beizutragen.

\section{Fazit für die Praxis}

Die wenigsten Kliniken behandeln nach eigenen Aussagen mehr als fünf Patienten mit retroperitonealem Sarkom im Jahr. Knapp die Hälfte der Kliniken behandelt Patienten ohne prätherapeutische histologische Sicherung, obwohl diese nach Datenlage hinsichtlich Komplikationen und Lokalrezidivrate unbedenklich ist. Eine multiviszerale Kompartmentresektion stellt die häufigste chirurgische Operationsstrategie dar. Eine regelhafte (neo-)adjuvante Therapie wird in der Mehrheit der Kliniken nicht durchgeführt. Insgesamt zeigt sich in Diagnostik und Therapie kein einheitliches Vorgehen bei retroperitonealen Sarkomen, was an der niedrigen Inzidenzrate und Behandlungsfrequenz sowie dem Fehlen einer nationalen S3Leitlinie liegen könnte. Die Mehrheit der Umfrageteilnehmer würde ein zentrales Behandlungsregister und gezielte Fortbildungsveranstaltungen unterstützen. Eine Zentrumsbildung zur Verbesserung der Behandlungsqualität erscheint sinnvoll.

\section{Korrespondenzadresse}

\section{PD Dr. J. Jakob}

Spezielle Chirurgische Onkologie und

Thoraxchirurgie, Chirurgische Klinik,

Universitätsmedizin Mannheim, Universität

Heidelberg

Theodor-Kutzer-Ufer 1-3, 68137 Mannheim, Deutschland

Jens.jakob@umm.de

Förderung. Die Open Access Publikation dieses Artikels wurde finanziert durch Das Lebenshaus e. V./ GISG/Deutsche Sarkom-Stiftung.

\section{Einhaltung ethischer Richtlinien}

Interessenkonflikt. J. Jakob, A. Gerres, U. Ronellenfitsch, L. Pilz, M. Wartenberg, B. Kasper, H.-R. Raab und $P$. Hohenberger geben an, dass kein Interessenkonflikt besteht.

Dieser Beitrag beinhaltet keine von den Autoren durchgeführten Studien an Menschen oder Tieren.

Open Access. Dieser Artikel wird unter der Creative Commons Namensnennung 4.0 International Lizenz (http://creativecommons.org/licenses/by/4.0/deed. de) veröffentlicht, welche die Nutzung, Vervielfältigung, Bearbeitung, Verbreitung und Wiedergabe in jeglichem Medium und Format erlaubt, sofern Sie den/die ursprünglichen Autor(en) und die Quelle ordnungsgemäßnennen, einen Linkzur Creative Commons Lizenz beifügen und angeben, ob Änderungen vorgenommen wurden.

\section{Literatur}

1. Ducimetiere F, Lurkin A, Ranchere-Vince $D$ et al (2011) Incidence of sarcoma histotypes and molecular subtypes in a prospective epidemiological study with central pathology review and molecular testing. PLOSONE 6:e20294

2. Mastrangelo $G$, Coindre JM, Ducimetiere F et al (2012) Incidence of soft tissue sarcoma and beyond: a population-based prospective study in 3 European regions. Cancer 118:5339-5348

3. Gronchi A, Strauss DC, Miceli R et al (2016) Variability in patterns of recurrence after resection of primary Retroperitoneal Sarcoma (RPS): a report on 1007 patients from the multi-institutional collaborative RPS working group. Ann Surg 263:1002-1009

4. Trans-AtlanticRPSWorking Group (2015) Management of primary retroperitoneal sarcoma (RPS) in the adult: a consensus approach from the TransAtlantic RPS Working Group. Annals of surgical oncology 22:256-263

5. von Mehren M, Randall RL, Benjamin RS etal (2016) Soft Tissue Sarcoma, Version 2.2016, NCCN Clinical Practice Guidelines in Oncology. J Natl Compr Canc Netw 14:758-786

6. BonvalotS, RautCP, Pollock REetal (2012) Technical considerations in surgery for retroperitoneal sarcomas: position paper from E-Surge, a master class in sarcoma surgery, and EORTC-STBSG. Ann Surg Oncol 19:2981-2991 
7. Storm FK, Mahvi DM (1991) Diagnosis and management of retroperitoneal soft-tissue sarcoma. Ann Surg 214:2-10

8. Smith MJ, Ridgway PF, Catton CN et al (2014) Combined management of retroperitoneal sarcoma with dose intensification radiotherapy and resection: long-term results of a prospective trial. Radiother Oncol 110:165-171

9. Bates JE, Dhakal S, Mazloom A, Constine LS (2015) The benefit of adjuvant radiotherapy in high-grade nonmetastatic retroperitoneal soft tissue sarcoma: a SEERanalysis. Am JClin Oncol. https://doi.org/10. 1097/coc.0000000000000259

10. Angele MK, Albertsmeier M, Prix NJ et al (2014) Effectiveness of regional hyperthermia with chemotherapy for high-risk retroperitoneal and abdominal soft-tissue sarcoma after complete surgical resection: a subgroup analysis of a randomized phase-III multicenter study. Ann Surg 260:749-754 (discussion 754-746)

11. Greenberg DD, Crawford B (2016) Surveillance strategies for sarcoma: results of a survey of members of the musculoskeletal tumor society. Sarcoma. https://doi.org/10.1155/2016/8289509

12. Sargent M, Boeck S, Heinemann V, Jauch KW, Seufferlein T, Bruns CJ (2011) Surgical treatment concepts for patients with pancreatic cancer in Germany - results from a national survey conducted among members of the "Chirurgische Arbeitsgemeinschaft Onkologie" (CAO) and the "Arbeitsgemeinschaft Internistische Onkologie" (AIO) of the Germany Cancer Society (DKG). Langenbeck's archives of surgery/Deutsche Gesellschaft fur. Chirurgie 396:223-229

13. Jakob J, Ronellenfitsch U, Wenz F, Marx A, Hohenberger $P$ (2014) Surgery of retroperitoneal soft tissue sarcomas and gastrointestinal stromal tumors. Onkologe 20:1088-1102

14. Scali EP, Chandler TM, Heffernan EJ, Coyle J, Harris AC, Chang SD (2015) Primary retroperitoneal masses: what is the differential diagnosis? Abdom Imaging 40:1887-1903

15. Berger-Richardson D, Swallow CJ (2016) Needle tract seeding after percutaneous biopsy of sarcoma: Risk/benefit considerations. Cancer. https://doi.org/10.1002/cncr.30370

16. Wilkinson MJ, Martin JL, Khan AA, Hayes AJ, Thomas JM, Strauss DC (2015) Percutaneous core needle biopsy in retroperitoneal sarcomas does not influence local recurrence or overall survival. Ann Surg Oncol 22:853-858

17. Doyle LA (2014) Sarcoma classification: an update based on the 2013 World Health Organization Classification of Tumors of Soft Tissue and Bone. Cancer 120:1763-1774

18. Baldini EH, Wang D, Haas RL et al (2015) Treatment guidelines for preoperative radiation therapy for retroperitoneal sarcoma: preliminary consensus of an international expert panel. Int J Radiat Oncol Biol Phys 92:602-612

19. Ray-Coquard I, Thiesse $P$, Ranchere-Vince $D$ et al (2004) Conformity to clinical practice guidelines, multidisciplinary management and outcome of treatment for soft tissue sarcomas. Ann Oncol 15:307-315

\section{Entwicklung modernster Navigationssysteme für die Gefäßchirurgie}

Bundesforschungsministerium fördert Gemeinschaftsprojekt Lübecker Chirurgen, Informatiker, Mathematiker und Laser-Wissenschaftler

Mit 4,2 Millionen Euro unterstützt das Bundesforschungsministerium ein Verbundprojekt am Standort Lübeck zur Entwicklung neuer Navigationssysteme für die Gefäßchirurgie. Beteiligt am Verbundprojekt „Kombinierte Navigation zur Endovaskulären Therapie an der Hauptschlagader" (Nav EVAR) sind die Klinik für Chirurgie des UKSH und das Universitäre Gefäßzentrum, das Institut für Robotik und Kognitive Systeme und die Fraunhofer MEVIS Projektgruppe für Bildregistrierung der Universität sowie das Medizinische Laserzentrum Lübeck.

In den westlichen Industrienationen stellen Gefäßerkrankungen, noch vor Tumorerkrankungen, die häufigste Todesursache dar. Hierzu zählen Erkrankungen der Herzkranzgefäße, Erkrankungen der Hirngefäße sowie Erkrankungen der Bauch- und Extremitätengefäße. Global weisen derzeit allein $202 \mathrm{Mil}$ lionen Menschen eine arterielle Erkrankung der Beingefäße auf. Neben der medikamentösen Therapie der Arteriosklerose erlangte durch den medizintechnischen Fortschritt der Katheterverfahren die minimal-invasive Therapie einen hohen Stellenwert in der Behandlung gefäßkranker Patienten.

Der Einsatz sogenannter endovaskulärer minimal-invasiver Verfahren wurde in den letzten zwei Jahrzehnten auf nahezu alle Gefäßabschnitte des Menschen ausgeweitet. Die notwendige Darstellung der Gefäßanatomie zur Steuerung der Katheter- und Therapiesysteme und zur Visualisierung der Gefäßpathologie wird in allen Verfahren und Fachgebieten (Kardiologie, Angiologie, Radiologie, Gefäßchirurgie, Neuroradiologie etc.) derzeit durch eine zweidimensionale Angiographie mit Kontrastmittelapplikation unter Röntgenstrahlung erreicht.

\section{Technologiekonzept Nav EVAR}

Das Technologiekonzept Nav EVAR könnte die vorhandenen Methoden möglicherweise vollständig ersetzen. Die Grundidee besteht darin, beispielsweise einen Stentgraft ohne Röntgenstrahlung und ohne Verwendung von Kontrastmitteln zur Gefäßdarstellung in der Hauptschlagader platzieren zu können. Im Fokus stehen dabei zwei Kernpunkte des
Verfahrens: die Reduktion der verfahrensspezifischen Risiken des bisherigen Verfahrens, insbesondere die Reduktion der Strahlenexposition für Patient und Behandler sowie die Reduktion der Kontrastmittelexposition des Patienten. Zweitens die verbesserte Visualisierung für den Behandler (durch erweiterte Realitätswahrnehmung, Augmented Reality) während des Eingriffes mit resultierender Qualitätssteigerung (mögliche Reduktion der Eingriffszeit, Reduktion von Prothesenfehlplatzierung, zusätzlicher Sicherheitsgewinn etc.).

Zur Umsetzung dieser Ziele ist die Kombination aus hardwarebasierten Nachführsystemen (Tracking, Sensorik) und modernster Bildgebungs- und Bildverarbeitungstechnologie notwendig. Im Rahmen des Projektes werden verschiedene Trackingsysteme in Fusion mit innovativer Visualisierungstechnologie untersucht und weiterentwickelt und sollen u.a. durch eine Ausgründung am Ende der Projektlaufzeit marktfähig gemacht werden.

Universität zu Lübeck

http://www.uni-luebeck.de 